\title{
Exercise order affects the total training volume and the ratings of perceived exertion in response to a super-set resistance training session
}

This article was published in the following Dove Press journal:

International Journal of General Medicine

10 February 2012

Number of times this article has been viewed

\author{
Sandor Balsamo ${ }^{1-3}$ \\ Ramires Alsamir Tibana ${ }^{1,2,4}$ \\ Dahan da Cunha \\ Nascimento ${ }^{1,2}$ \\ Gleyverton Landim de \\ Farias $^{1,2}$ \\ Zeno Petruccelli ${ }^{1,2}$ \\ Frederico dos Santos de \\ Santana ${ }^{1,2}$ \\ Otávio Vanni Martins ${ }^{1,2}$ \\ Fernando de Aguiar ${ }^{1,2}$ \\ Guilherme Borges Pereira ${ }^{4}$ \\ Jéssica Cardoso de Souza ${ }^{4}$ \\ Jonato Prestes ${ }^{4}$ \\ 'Department of Physical Education, \\ Centro Universitário UNIEURO, \\ Brasília, ${ }^{2}$ GEPEEFS (Resistance \\ training and Health Research Group), \\ Brasília/DF, ${ }^{3} \mathrm{Graduate}$ Program \\ in Medical Sciences, School of \\ Medicine, Universidade de Brasília \\ $(U n B)$, Brasília, ${ }^{4}$ Graduation Program \\ in Physical Education, Catholic \\ University of Brasilia (UCB), Brasília/ \\ DF, Brazil
}

Correspondence: Sandor Balsamo Programa de Pós-Graduação em Ciências Médicas, Faculdade de Medicina, Universidade de Brasília, UnB, CEP: 70910-900, Brasília/DF, Brazil

Tel +55613107 I70|

$\mathrm{Fax}+55613107 \quad 1907$

Email sandorbalsamo@gmail.com

\begin{abstract}
The super-set is a widely used resistance training method consisting of exercises for agonist and antagonist muscles with limited or no rest interval between them - for example, bench press followed by bent-over rows. In this sense, the aim of the present study was to compare the effects of different super-set exercise sequences on the total training volume. A secondary aim was to evaluate the ratings of perceived exertion and fatigue index in response to different exercise order. On separate testing days, twelve resistance-trained men, aged $23.0 \pm 4.3$ years, height $174.8 \pm 6.75 \mathrm{~cm}$, body mass $77.8 \pm 13.27 \mathrm{~kg}$, body fat $12.0 \% \pm 4.7 \%$, were submitted to a super-set method by using two different exercise orders: quadriceps (leg extension) + hamstrings (leg curl) $(\mathrm{QH})$ or hamstrings (leg curl) + quadriceps (leg extension) (HQ). Sessions consisted of three sets with a ten-repetition maximum load with 90 seconds rest between sets. Results revealed that the total training volume was higher for the HQ exercise $\operatorname{order}(P=0.02)$ with lower perceived exertion than the inverse order $(P=0.04)$. These results suggest that HQ exercise order involving lower limbs may benefit practitioners interested in reaching a higher total training volume with lower ratings of perceived exertion compared with the leg extension plus leg curl order.
\end{abstract}

Keywords: resistance training, super-set method, ratings of perceived exertion, total training volume

\section{Introduction}

Muscle strength has an important influence on functional abilities, such as walking, stair climbing, and daily activities, as well as positive association with sports performance, ${ }^{1}$ longevity, and quality of life. ${ }^{2-4}$ Nevertheless, the progression of resistance training relies on the interplay between volume, intensity and training methods.

The super-set is a widely used method consisting of exercises for agonist and antagonist muscles with limited or no rest interval between them - for example, bench press followed by lateral pull-down. ${ }^{5}$ This training strategy has been recently mentioned by the American College of Sports Medicine ${ }^{1}$ as one in which the strength and power of the upper body can be optimized with agonist and antagonist exercises. ${ }^{6,7}$ However, Maynard and Ebben ${ }^{8}$ found a decrease in lower limb torque and electromyography with antagonist prefatigue. Alternatively, Kaminura and Takenaka ${ }^{9}$ showed that the preactivation of the hamstrings (antagonist muscles) resulted in improved torque of the quadriceps (agonist muscles) in the isokinetic equipment.

Thus, results regarding the effects of previous studies are rather controversial and require further investigation. ${ }^{10}$ Several studies have used isokinetic equipment, but the comparison with usual free weights and machines used in gyms and training facilities 
is difficult. ${ }^{6,11}$ Although previous studies have tested the super-set method as an exercise protocol to evaluate torque and power, no study has analyzed the effects of altering muscle-use order during super-sets for the lower limbs with typical isoinertial resistance training machines.

Furthermore, related findings have been equivocal regarding exercise sequence and training volume. For example, Sforzo and Touey ${ }^{12}$ found that total training volume is superior when training is initiated with large muscle groups followed by small muscle groups. However, other studies reported either no differences in training volume, regardless of the exercise order, when using the pre-exhaustion method or found that a higher volume of training could be performed when small muscle groups were used first. ${ }^{13,14}$ This reinforces the idea that exercise order in super-set method will affect training volume.

Therefore, the primary aim of the present study was to compare the effect of the super-set method performed in different muscle orders on the total training volume. A secondary aim was to evaluate the ratings of perceived exertion (RPE) in response to different exercise order by using the OMNI scale (OMNI-Resistance Exercise Scale-OMINI-RES; 0 = resting condition, $10=$ maximum effort).${ }^{15}$ The initial hypothesis was that in the exercise order hamstrings + quadriceps (HQ) the total training volume would be higher and the RPE lower than the inverse order quadriceps + hamstrings $(\mathrm{QH})$.

\section{Methods}

\section{Experimental approach}

Twelve trained men participated in this study and visited the laboratory on five occasions. The subjects participated in a familiarization session as well as a ten-repetition maximum (RM) test on two different days separated by 48-72 hours to determine test re-test reliability. The same individuals were randomly submitted to a super-set method using two different exercise orders: QH or HQ separated by 48-72 hours. In the super-set method used in the present study, two exercises (agonist and antagonist) were executed with no rest interval between them. The dependent variables were the total training volume and the RPE. The independent variables were the two different exercise orders: QH and HQ.

\section{Subjects}

Twelve men aged $23.0 \pm 4.3$ years, height $174.8 \pm 6.75 \mathrm{~cm}$, body mass $77.8 \pm 13.27 \mathrm{~kg}$, body fat $12.0 \% \pm 4.7 \%$, participated in the study. Subjects had a minimum 6 months of previous experience with resistance training and trained 2-4 times per week using loads of 6-15 RM in sessions lasting up to 45-60 minutes. According to the American
College of Sports Medicine, ${ }^{1}$ therefore, the individuals were considered "trained." Exclusion criteria included: use of ergogenic supplements, steroid hormones, medications, and the presence of any type of cardiopulmonary disease or orthopedic limitation. Information regarding the benefits, risks, and nature of the study were provided. The subjects were advised to refrain from ingesting caffeine and alcohol for 24 hours before all tests, avoid any strenuous exercise in the 48-hours before the experimental sessions, and to maintain their normal daily diet during the study, according to the authors' previous study. ${ }^{16}$ Before data collection, volunteers completed the Physical Activity Readiness Questionnaire ${ }^{17}$ and signed an informed consent document approved by the Euro-American University Center Research Ethics Committee for Humans (Protocol no. 056/2009).

\section{Strength testing}

A familiarization session was carefully performed to allow for correct execution of the leg extension and leg curl exercise technique. After 48-72 hours, the 10 RM test was performed on two different days separated by 48-72 hours to guarantee optimal test retest reliability. ${ }^{18}$ Before the initiation of the 10 RM test, a warm-up of two sets with submaximal loads for each exercise was allowed. After a rest interval of 2-4 minutes, individuals performed the first attempt and the load was increased until the determination of the $10 \mathrm{RM}$. No more than five attempts were used to determine the $10 \mathrm{RM}$ for leg extension and leg curl in isoinertial machines (Leg Extension, SL153: quadriceps and seated leg curl, SL160: biceps femoris and calf; Johnson Health Technologies Inc, Taichung Hsien, Taiwan). The $10 \mathrm{RM}$ tests were randomly chosen and a minimal 10-minute rest interval was allowed between the tests.

Briefly, to minimize error, the following strategies were adopted: standardized instructions concerning the testing procedure were given to the participants before the test; participants received standardized instructions about exercise technique; body position was held constant; and verbal encouragement was provided during the testing procedure. The detailed description of the initial position and concentric phase of each exercise is presented below. The eccentric action of both exercises was performed after the final phase of the concentric action.

- Leg extension: seated position in the machine with $90^{\circ}$ knee flexion and arms positioned toward the body by holding the fixed device support; the concentric phase was performed from the initial position until full knee extension. 
- Leg curl: seated position with $180^{\circ}$ knee flexion and arms positioned toward the body by holding the fixed device support; the concentric phase was performed from the initial position until $90^{\circ}$ knee flexion was achieved.

\section{Super-set training session}

Two days after the $10 \mathrm{RM}$ tests, the super-set training sessions were randomly performed with $48-72 \mathrm{~h}$ of rest interval between them: the $\mathrm{QH}$ super-set session (= leg extension + leg curl order) and the HQ session (= leg curl + leg extension order). Before initiation of the superset sessions, a warm-up of two sets of twelve repetitions at $40 \%$ of $10 \mathrm{RM}$ was allowed with a 90 -second rest interval after them. Subjects performed three sets until voluntary concentric failure with $10 \mathrm{RM}$ loads and a rest interval of 90 seconds between sets. RPE was verified with the OMNI scale designed for resistance training immediately after each set in both exercise orders. ${ }^{11}$ No attempt was made to control the movement velocity during each repetition of the exercises. ${ }^{18}$ All sessions were supervised individually by an experienced resistance-training professional.

\section{Total training volume calculation}

The calculation of the total training volume for each exercise order was made by using the following equation: training volume $1+$ training volume $2+$ training volume 3 . The training volume was calculated as: number of repetitions $\times$ load. The fatigue index, commonly defined as the drop in strength and power during a training session, was estimated for each exercise in both orders using the formula proposed by Dipla et $\mathrm{al}^{19}: \mathrm{FI}=($ third set/first set $) \times 100$; where a higher percentage value $(\%)$ indicates a superior fatigue resistance.

\section{Statistical analysis}

Reliability of the 10 RM tests was accessed by the intraclass correlation test and the values were 0.91 and 0.93 for the leg extension and leg curl, respectively. The ShapiroWilk normality test and a homoscedasticity test (Bartlett's criterion) were used to test the normal distribution of the data. All variables presented a normal distribution and homoscedasticity. The comparison between both super-set exercise orders (QH and HQ) in each set was accessed by the two-entries analysis of variance test (orders $\times$ sets). The Bonferroni post hoc test was applied where indicated by an analysis of variance. To verify the differences in the total training volume and RPE between $\mathrm{QH}$ and HQ exercise orders, the unpaired Student's $t$-test and Wilcoxon test were used, respectively. In all calculations, the alpha level was set at $P \leq 0.05$. SPSS for Windows (v 16.0; SPSS, Inc, Chicago, IL) was used for all analyses. The effect size was calculated according the classification proposed by $\mathrm{Rhea}^{20}$ for trained individuals $(<0.35=$ insignificant, $0.35-0.80=$ small, $0.50-1.50=$ moderate, $>1.5=$ high $)$.

\section{Results}

Table 1 presents the values of training volume performed in each set and the total training volume for all three sets for both exercise orders. Training volume was significantly lower for the second and third sets compared with the first set for both QH and HQ order. Additionally, in the second and third sets of $\mathrm{QH}$, training volume was lower than in the second and third sets of HQ order. Regarding the total training volume, the values were lower in the $\mathrm{QH}$ order compared with the HQ $(P=0.02)$ (Table 1 and Figure 1$)$. Conversely, RPE was higher in the $\mathrm{QH}$ order compared with HQ order $(P=0.04)$ (Figure 2). Table 2 presents effect sizes for the three sets and for the total training volume. Results revealed a superior moderate effect size in set 2 and 3 , and total training volume for the HQ super-set order compared with the QH order.

\section{Discussion}

The main findings of the present study revealed that when a super-set method was initiated with the HQ order (leg curl + leg extension) an increased total training volume could be achieved with a lower RPE compared with the $\mathrm{QH}$ order (leg extension + leg curl). Considering this, the initial hypothesis is confirmed, since, in the leg curl preceding order, individuals presented a higher muscle performance.

It has been proposed that a preceding stimulation of the antagonist flexor muscle and its superior effect on the total training volume can be mediated by neural adjustment

Table I Total volume completed in each set and fatigue index for both super-set exercise orders

\begin{tabular}{lllll}
\hline Order & Set I & Set 2 & Set 3 & Fatigue index (\%) \\
\hline $\mathrm{QH}$ & $2126.83 \pm 347.85$ & $1307.67 \pm 277.35^{*}$ & $923.67 \pm 298.96 *$ & $44.00 \pm 14.80$ \\
$\mathrm{HQ}$ & $2298.42 \pm 475.94$ & $1583.50 \pm 321.63^{*}$ & $1226.33 \pm 325.09 * . \neq$ & $54.50 \pm 15.50$ \\
\hline
\end{tabular}

Notes: Values are means \pm standard deviation of the mean. *Statistically significant difference from set $I$; ₹statistically significant different from set 2 ( $P \leq 0.05$ ). Abbreviations: QH, quadriceps (leg extension) + hamstrings (leg curl) super-set exercise order; HQ, hamstrings (leg curl) + quadriceps (leg extension) super-set exercise order. 


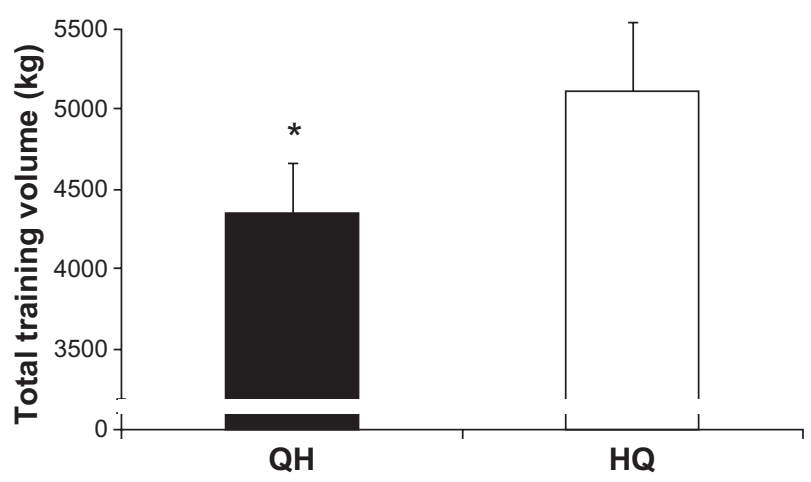

Figure I Total training volume for the $\mathrm{QH}=$ leg extension + leg curl super-set exercise order and $\mathrm{HQ}=$ leg curl + leg extension super-set exercise order.

Notes: *Difference between $\mathrm{QH}$ and $\mathrm{HQ}$ exercise order $(P \leq 0.05)$. Values are means \pm standard deviation of the mean.

Abbreviations: $\mathrm{QH}$, quadriceps (leg extension) + hamstrings (leg curl) super-set exercise order; HQ, hamstrings (leg curl) + quadriceps (leg extension) super-set exercise order.

(Golgi tendon organ) allowing muscle actions to become more intense, ${ }^{21}$ elastic energy, ${ }^{8}$ or by an alteration on the triphasic neural pathway, suggested as a possible mechanism responsible for performance enhancement. ${ }^{6}$ Apart from this, Aagaard et $\mathrm{al}^{22}$ observed that antagonist hamstring movements counteract the anterior tibial shear and excessive internal tibial rotation induced by the contractile forces of the quadriceps near full knee extension. However, it has been shown that antagonist activation may not affect the performance of a standard isokinetic fatigue test. ${ }^{10}$ Thus, the decrease in the resultant joint moment after fatigue could be attributed to changes in agonist (knee extensor) muscle force-generation capacity rather than an altered moment of force exerted by

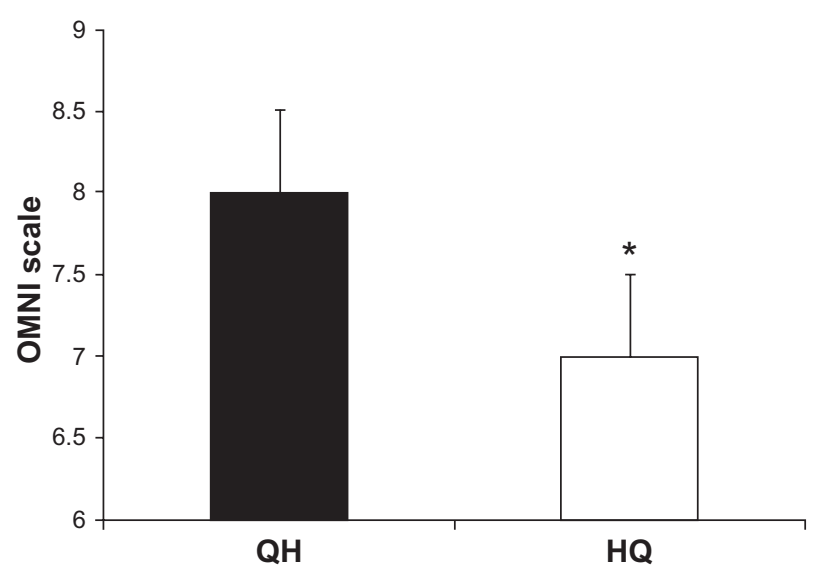

Figure 2 Ratings of perceived exertion determined by the OMNI scale for the $\mathrm{QH}=$ leg extension + leg curl super-set exercise order and $\mathrm{HQ}=$ leg curl + leg extension super-set exercise order.

Notes: *Difference between $\mathrm{QH}$ and HQ exercise order $(P \leq 0.05)$. Values are median.

Abbreviations: $\mathrm{QH}$, quadriceps (leg extension) + hamstrings (leg curl) super-set exercise order; HQ, hamstrings (leg curl) + quadriceps (leg extension) super-set exercise order.
Table 2 Values of effect size (ES) for the total volume performed in each set

\begin{tabular}{lllll}
\hline Set & QH & HQ & ES & P \\
\hline Set I & $2126.83 \pm 347.85$ & $2298.42 \pm 475.94$ & 0.52 & 0.3 \\
Set 2 & $1307.67 \pm 277.35$ & $1583.50 \pm 321.63 *$ & 0.99 & 0.03 \\
Set 3 & $923.67 \pm 298.96$ & $1226.33 \pm 325.09 *$ & 1.01 & 0.02 \\
\hline
\end{tabular}

Notes: Values are means \pm standard deviation of the mean. $\mathrm{QH}=$ leg extension + leg curl super-set exercise order and HQ = leg curl + leg extension super-set exercise order. *Difference between $\mathrm{QH}$ and $\mathrm{HQ}$ exercise order $(P \leq 0.05)$.

Abbreviations: $\mathrm{QH}$, quadriceps (leg extension) + hamstrings (leg curl) super-set exercise order; HQ, hamstrings (leg curl) + quadriceps (leg extension) super-set exercise order.

the antagonist (hamstrings). ${ }^{23}$ Nevertheless, the exact neural mechanisms of flexor antagonist stimulation prior to knee extension remain to be elucidated in future studies.

The limitations of the present study are that no eletromyographic measures were made and the small, male-only, sample, which may limit the external validity of these findings.

An interesting feature of the present study is the use of conventional resistance training machines, since only a few studies using isokinetic devices investigated the effect of a super-set method involving the interchange between agonist and antagonist muscles of the lower limb. ${ }^{8,9}$ Although little has been reported on this phenomenon, it is known that exercise order can acutely affect muscle strength. ${ }^{12}$ Baker and Newton ${ }^{6}$ showed that muscle and power were increased by the previous use of the antagonist muscle for the upper body, highlighting the benefits of the super-set method for the upper body. However, when isokinetic equipment was used, the prefatigue of the antagonist muscle resulted in a reduced torque of the quadriceps at $60^{\circ} . \mathrm{s}-1$, suggesting a limitation of the super-set method for the lower body. ${ }^{8}$

Alternatively, the results of the present study revealed that the super-set method was more effective for the total training volume by using the pre-activation of the hamstrings (leg curl) compared with the order that was initiated with the quadriceps (leg extension). The calculation of the effect size reinforced the above mentioned results with a moderate effect (between 0.50 and 1.50) favoring the HQ order. These results are different from those studies that used isokinetic machines, which are not commonly used in the daily practice of resistance training. In this sense, adding to the benefits of the super-set method on energy expenditure and time optimization in a resistance training session, ${ }^{5}$ this method can be effective in increasing total training volume when leg curl precedes leg extension. Kraemer and Ratamess ${ }^{24}$ indicated that total training volume is an important variable in muscle hypertrophy.

Another interesting result was that the RPE was lower in the leg curl + leg extension order. The use of the leg extension 
(quadriceps) first will exacerbate the RPE in a super-set method compared with the inversed order. It has been shown that the RPE can change with different resistance training volume. ${ }^{25,26}$ However, these explanations are rather speculative and require further investigation. The interchange between agonist and antagonist muscles possible when using a super-set method with conventional resistance training equipment is a promising area of research for investigators and resistance training professionals. Future studies using the super-set method should be carried out with acute and chronic designs and different exercise orders in different muscle groups and individuals, analyzing muscle hypertrophy, strength, and hormonal response, and with the use of electromyography.

\section{Acknowledgments}

The authors would like to thank Hildeamo Bonifácio $\mathrm{PhD}$ for statistics contributions and the members of the GEPEEFS (Resistance training and Health Research Group): Daniel Tavares, Daiane Priscila Dantas da Silva, and Igor Magalhães.

\section{Disclosure}

The authors declare no conflicts of interest in this work.

\section{References}

1. American College of Sports Medicine. Position stand on progression models in resistance training for healthy adults. Med Sci Sports Exerc. 2009;41:687-708.

2. Newman AB, Kupelian V, Visser M, et al. Strength, but not muscle mass, is associated with mortality in the health, aging and body composition study cohort. J Gerontol A Biol Sci Med Sci. 2006;61:72-77.

3. Visser M, Goodpaster BH, Kritchevsky SB, et al. Muscle mass, muscle strength, and muscle fat infiltration as predictors of incident mobility limitations in well-functioning older persons. J Gerontol A Biol Sci Med Sci. 2006;60:324-333.

4. Balsamo S, Santos-Neto LD. Fatigue in systemic lupus erythematosus: an association with reduced physical fitness. Autoimmun Rev. 2011;10: 514-518.

5. Kelleher AR, Hackney KJ, Fairchild TJ, Keslacy S, Ploutz-Snyder LL. The metabolic costs of reciprocal supersets vs traditional resistance exercise in young recreationally active adults. J Strength Cond Res. 2010; 24:1043-1051.

6. Baker D, Newton RU. Acute effect on power output of alternating an agonist and antagonist muscle exercise during complex training. J Strength Cond Res. 2005;19:202-205.

7. Burke DG, Pelham TW, Holt LE. The influence of varied resistance and speed of concentric antagonistic contractions on subsequent concentric agonistic efforts. J Strength Cond Res. 1999;13:193-197.
8. Maynard J, Ebben WP. The effects of antagonist pre-fatigue on agonist torque and electromyography. J Strength Cond Res. 2003;17: 469-474.

9. Kaminura T, Takenaka T. Potentiation of knee extensor contraction by antagonist conditioning contraction at several intensities. $J$ Physiol Anthropol. 2007;26:443-447.

10. Bohannon RW, Gibson DF, Larkin, P. Effect of resisted knee flexion on knee extension torque. Phys Ther. 1986;66:1239-1241.

11. Robertson RJ, Goss FL, Rutkowski J, et al. Concurrent validation of the OMNI perceived exertion scale for resistance exercise. Med Sci Sports Exer. 2003;35:333-341.

12. Sforzo GA, Touey PR. Manipulating exercise order affects muscular performance during a resistance exercise training session. J Strength Cond Res. 1996;10:20-24.

13. Gentil P, Oliveira E, de Araújo Rocha Júnior V, do Carmo J, Bottaro M. Effects of exercise order on upper-body muscle activation and exercise performance. J Strength Cond Res. 2007;21:1082-1086.

14. Bellezza PA, Hall EE, Miller PC, Bixby WR. The influence of exercise order on blood lactate, perceptual, and affective responses. J Strength Cond Res. 2009;23:203-208.

15. Lagally KM, Robertson RJ. Construct validity of the OMNI resistance exercise scale. J Strength Cond Res. 2006;20:252-256.

16. Tibana RA, Prestes J, da Cunha Nascimento D, Martins OV, De Santana FS, Balsamo S. Higher Muscle Performance in Adolescents Compared With Adults After a Resistance Training Session With Different Rest Intervals. J Strength Cond Res. 2011. Epub August 30, 2011.

17. Shephard RJ. PAR-Q, Canadian Home fitness test and exercise screening alternatives. Sports Med. 1998;5:185-195.

18. Simão R, Farinatti Pde T, Polito MD, Maior AS, Fleck SJ. Influence of exercise order on the number of repetitions performed and perceived exertion during resistance exercises. J Strength Cond Res. 2005;19: $152-156$.

19. Dipla K, Tsirini T, Zafeiridis A, et al. Fatigue resistance during highintensity intermittent exercise from childhood to adulthood in males and females. Eur J Appl Physiol. 2009;106:645-653.

20. Rhea M. Determining the magnitude of treatment effects in strength training research through the use of the effect size. J Strength Cond Res. 2004;18:918-920.

21. Fleck SJ, Kraemer WJ. Designing Resistance Training Programs. Champaign, IL: Human Kinetics, 2004.

22. Aagaard P, Simonsen EB, Andersen JL, Magnusson SP, Bojsen-Møller F, Dyhre-Poulsen P. Antagonist muscle coactivation during isokinetic knee extension. Scand J Med Sci Sports. 2000;10:58-67.

23. Kellis E. Antagonist moment of force during maximal knee extension in pubertal boys: effects of quadriceps fatigue. Eur J Appl Physiol. 2003; 89:271-80.

24. Kraemer WJ, Ratamess N. Hormonal responses and adaptations to resistance exercise and training. Sports Med. 2005;35:339-361.

25. Sweet TW, Foster C, McGuigan MR, Brice G. Quantification of resistance training using the session rating of perceived exertion method. J Strength Cond Res. 2004;18:796-802.

26. Tiggemann CL, Korzenowski AL, Brentano MA, Tartaruga MP, Alberton CI, Kruel LFM. Perceived exertion in different strength exercise loads in sedentary, active, and trained adults. J Strength Cond Res. 2010;24:2032-2041.
International Journal of General Medicine

\section{Publish your work in this journal}

The International Journal of General Medicine is an international, peer-reviewed open-access journal that focuses on general and internal medicine, pathogenesis, epidemiology, diagnosis, monitoring and treatment protocols. The journal is characterized by the rapid reporting of reviews, original research and clinical studies across all disease areas.

\section{Dovepress}

A key focus is the elucidation of disease processes and management protocols resulting in improved outcomes for the patient.The manuscript management system is completely online and includes a very quick and fair peer-review system. Visit http://www.dovepress.com/ testimonials.php to read real quotes from published authors. 